\title{
Coupling Analysis of ECG and EMG based on Multiscale symbolic transfer entropy
}

\author{
Lizhao Du', a , Wenpo Yao, b, Jun Wang ${ }^{3, c}$ \\ School of Geographic and Biologic information, Nanjing Univ. of Posts \& Telecomm, Nanjing, China \\ a18251955996@163.com, ${ }^{\mathrm{b}}$ yaowen512@163.com, ${ }^{\mathrm{c} w a n g j @ n j u p t . e d u . c n ~}$
}

Keywords: Multiscale symbolic transfer entropy, ECG, EMG

\begin{abstract}
It has long been the focus of many scholars at home and abroad to explore the coupling of dynamic systems. However, the coupling analysis of basic transfer entropy cannot effectively determine the coupling situation in a certain range, and therefore, based on the research of original transfer entropy, we use multiscale symbolic transfer entropy(MSTE) to analyze it and only find that the multi-scale symbol transfer entropy value of the coupling analysis of ECG and EMG corresponds to different distribution trends at different scales. Later, we find out the best signal data extraction method and use it in our analysis. The experiment result shows that the value of it in the sleep time is larger than awake period, which means that the coupling of ECG and EMG is more significant in the sleep time, and we can capture the dynamic information in the signal and the change in system dynamics complexity more easily, which is better for the application of medical clinical practice.
\end{abstract}

\section{Introduction}

The research of various kinds of bioelectricity in human body has been an important means of understanding the function, clinical diagnosis and treatment of various organs. ECG(electrocardiogram), reflects the electrical changes in the production, conduction, and recovery of the heart while EMG(electromyography) determines the functional state of the neuromuscular unit by describing the biological current of the activity of the neuromuscular unit. All these parameters are closely related to the physiological state of the body. The application of ECG and EMG in clinical practice is of great importance. Since the 1990's, the nonlinear dynamic method is applied to the study of physiological electrical signals and makes some progress. ECG and EMG are very complicated non-stationary signals and therefore, comparing with other bio-electricity signals, the analysis technics of them need further to be developed. [1-3] So far, the main analytical methods are time-domain frequency domain analysis[4-9], neural network[9-14], chaotic analysis[15-17], etc, which represents the research direction of modern ECG and EMG. From physics category to neuroscience research, since synchronization and related phenomena of complex interactions are detected, trying to explore in the power system coupling relationship has always been a hot topic of many scholars at home and abroad. In recent years, the investigation and research in relevant fields mainly involve two aspects: the detection and quantification of the coupling strength and direction. Above all, the time sequence symbolization analysis is a method developed by the theory of symbolic dynamics and chaotic sequence analysis[18-19], which actually is to convert the original time series into a sequence of symbols, and then analyze further . Traditional symbolic dynamics methods are used to divide the region of symbols using a static range, and during the process of symbolization, the original sequence loses some of the details. Although the dynamics are preserved, the consequences will be severe affected because of the unsmoothness of the sequence. In respect of the issues above, we use multiscale symbolic transfer entropy(MSTE) to analyze the coupling of ECG and EMG and only find that the multi-scale symbol transfer entropy value of it corresponds to different distribution trends at different scales. By using this method to calculate the quantification of the coupling of the multi-scale symbol transfer entropy of each other through the use of the 
signals of the waking period and the sleeping period, and factors such as coupling strength and quantification are analyzed, we can capture the dynamic information in the signal and the change in system dynamics complexity more easily, which is better for the application of medical clinical practice.

\section{Transfer entropy, symbolic transfer entropy and Multiscale symbolic transfer entropy}

\subsection{Transfer entropy}

We usually use mutual information when describing the information interaction between two relational systems. However, the mutual information does not contain kinetics and directionality information. In order to study the dynamics and directional information of two systems sharing information, Thomas Schreiber proposed the concept of transfer entropy in 2000.

Let, $\quad x_{-} i=x(i), y_{i}=y(i), \mathrm{i}=1, \ldots, \mathrm{N}$, denote sequences of observations from systems $\mathrm{X}$ and Y. Transfer entropy incorporates time dependence by relating previous samples $x_{i}$ and $y_{i}$ to predict the next value $y_{i+1}$ and quantifies the deviation from the generalized Markov property, $\mathrm{p}\left(x_{i+1} \mid x_{i}, y_{i}\right)=\mathrm{p}\left(x_{i+1} \mid x_{i}\right)$, where $\mathrm{p}$ denotes the transition probability density. If there is no deviation from the generalized Markov property, Y has no influence on X. Transfer entropy, which is formulated as permutation entropy between $\mathrm{p}\left(x_{i+1} \mid x_{i}, y_{i}\right)$ and $\mathrm{p}\left(x_{i+1} \mid x_{i}\right)$, is defined as follows:

$$
\mathrm{T}_{\mathrm{X} \rightarrow \mathrm{Y}}=\sum \mathrm{p}\left(\mathrm{x}_{\mathrm{n}+1}, \mathrm{x}_{\mathrm{n}}^{(\mathrm{k})}, \mathrm{y}_{\mathrm{n}}^{(\mathrm{l})}\right) \log \frac{\mathrm{p}\left(\mathrm{x}_{\mathrm{n}+1} \mid \mathrm{x}_{\mathrm{n}}^{(\mathrm{k})}, \mathrm{y}_{\mathrm{n}}^{(\mathrm{l})}\right)}{\mathrm{p}\left(\mathrm{x}_{\mathrm{n}+1} \mid \mathrm{y}_{\mathrm{n}}^{(\mathrm{l})}\right)}
$$

Where $x_{n}$ and $y_{n}$ respectively represents the state of $\mathrm{X}$ and $\mathrm{Y}$ in a given time $\mathrm{n}, x_{n}^{(k)}$ means $\left(x_{n}, \ldots, x_{n-k+1}\right)$, usually $\mathrm{k}=1=1$.

Since the concept of transfer entropy is brought up, a series of calculations is used in transfer entropy calculating. However, most of them have higher requirements for observational data. Therefore, it is necessary to do preprocessing for the original data. This is what we are going to do in the next paragraph.

\subsection{Symbolic transfer entropy}

Symbolic dynamics, an abstract mathematical theory, developed in the 1920's, has become more and more popular with in-depth study of chaos, and become a powerful tool for analyzing chaotic phenomena and complex sequences. By splitting the states in dynamics into the appropriate parts and then get a certain symbol sequence, Symbolic dynamics is to analyze dynamics systems. It is the abstract and coarse grain of the complex dynamical system.

A method based on symbolic time series is to transform the original time sequence into a series of that consists of some symbols and then analyze this coarse grain algorithm. During the process of symbolization, the original sequence loses some of the details, though, the dynamic characteristics of the time sequence is reserved. And in this way, one of the greatest strengths is that we can improve the speed of calculating. Let $\mathrm{x}=\left\{x_{i}, 1 \leq i \leq N\right\}$, where $\mathrm{x}$ means the time sequence extracted, the length is N. Sequence reconstruction is the first step of permutation entropy as shown in Eq. 2, where $\mathrm{m}$ is embedding dimensions and $\tau$ is delay factor.

$X(i)=[x(i), x(i+\tau), \ldots, x(i+(m-1) \tau)], i=1,2, \ldots, N-m+1$

After the sequence reconstruction, we have gotten a $(\mathrm{N}-\mathrm{m}+1) \times \mathrm{m}$ matrix, which has $\mathrm{N}-\mathrm{m}+1$ $\mathrm{m}$-dimensional vectors. And then, for each of it as X(i), we calculate the base scale BS, which is the root-mean-square of the differences between every two contiguous data points in an $\mathrm{m}$-dimensional vectors.

$\mathrm{BS}(\mathrm{i})=\sqrt{\frac{\sum_{j=1}^{m-1}\left((x(i+j)-x(i+j-1))^{2}\right.}{m-1}}$

Based on the base scale, the partition standard can be selected as $\alpha \times$ BS(i), we transform each 
m-dimensional vector $X(i)$ into symbolic sequences

$S_{i}(\mathrm{i})=\{\mathrm{s}(\mathrm{i}), \mathrm{s}(\mathrm{i}+1), \ldots, \mathrm{s}(\mathrm{i}+\mathrm{m}-1)\}, \mathrm{i}=1,2, \ldots, \mathrm{N}-\mathrm{m}+1, \quad \tau=1, \quad \mathrm{~s} \in \mathrm{L}(\mathrm{L}=0,1,2,3)$. The specific symbolic process is as follow:

$S_{i}(\mathrm{i})\left\{\begin{array}{cc}0: & \bar{x}<x_{i+k} \leq \bar{x}+\alpha \times \mathrm{BS}(\mathrm{i}), \\ 1: & x_{i+k}>\bar{x}+\alpha \times \mathrm{BS}(\mathrm{i}), \\ 2: & \bar{x}-\alpha \times \mathrm{BS}(\mathrm{i})<x_{i+k} \leq \bar{x}, \\ 3: & x_{i+k} \leq \bar{x}-\alpha \times \mathrm{BS}(\mathrm{i}),\end{array}\right.$

Where $\mathrm{i}=1,2,3, \ldots, \mathrm{N}-\mathrm{m}+1, \mathrm{k}=0,1,2, \ldots, \mathrm{m}-1$ and $\mathrm{m}$ is choosing from 3 to $7, \mathrm{~N}$ is required to be larger than $4 \mathrm{~m} . \bar{x}$ and $\mathrm{BS}(\mathrm{i})$ respectively denotes the mean and the base scale of the ith m-dimensional vector $\mathrm{X}(\mathrm{i})$. The symbols $0,1,2$, and 3 are used only as a mark for each region, whose values have no practical meaning. $\alpha$ is a special parameter. If $\alpha$ is too large during transforming original time series to symbolic time series, the detail information will be lost and the dynamic information can not be caught. Too small an $\alpha$ value will result in salient influence from noise. We can see it in the Fig. 1

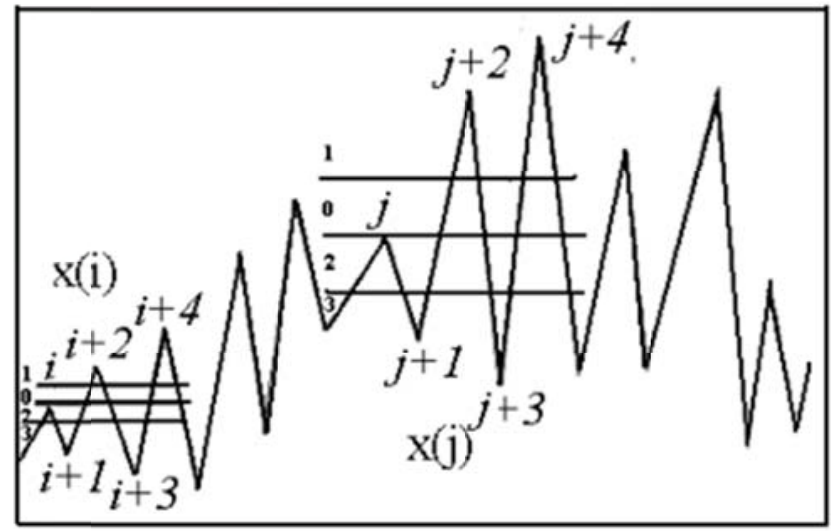

Fig. 1 the symbolization of time series

After the symbolization of time series, we extract the information of the sequence by coding adjacent symbols, and deem the probability that the code word appears in the entire time sequence as the analyzing parameter.

$h_{X Y}=-\sum p\left(x_{t+1}, x_{t}, y_{t}\right) \cdot \log p\left(x_{t+1} \mid x_{t}, y_{t}\right)$

$h_{x y}^{\prime}=-\sum p\left(x_{t+1}, x_{t}, y_{t}\right) \cdot \log p\left(x_{t+1} \mid x_{t}\right)$

$S T E_{X \rightarrow Y}=h_{x y}^{\prime}-h_{X Y}=\sum p\left(x_{t+1}, x_{t}, y_{t}\right) \cdot \log \frac{p\left(x_{t+1} \mid x_{t}, y_{t}\right)}{p\left(x_{t+1} \mid x_{t}\right)}$

Due to

$p\left(x_{t+1} \mid x_{t}, y_{t}\right)=\frac{p\left(x_{t+1}, x_{t}, y_{t}\right)}{p\left(x_{t}, y_{t}\right)}$

$p\left(x_{t+1} \mid x_{t}\right)=\frac{p\left(x_{t}, x_{t+1}\right)}{p\left(x_{t}\right)}$

Thus

$S T E_{X \rightarrow Y}=\sum p\left(x_{t+1}, x_{t}, y_{t}\right) \cdot \log \frac{p\left(x_{t+1}, x_{t}, y_{t}\right) p\left(x_{t}\right)}{p\left(x_{t}, y_{t}\right) p\left(x_{t}, x_{t+1}\right)}$ 


\subsection{Multiscale symbolic transfer entropy}

Costa[22] and his team proposed Multiscale entropy (MSE) as a method to deal with complex physiological time sequences. The algorithm is :

Given a one dimensional discrete time sequence, $\left\{x_{1}, \ldots, x_{i}, \ldots, x_{N}\right\}$, we build a serialized sequence of time $\left\{y^{\tau}\right\}$, determined by scale factor $\tau$,

$y_{j}^{(\tau)}=1 / \tau \sum_{i=(j-1) \tau+1}^{j \tau} x_{i}, 1 \leq \mathrm{j} \leq N / \tau$

As $\tau$ is 1 , it means $\left\{y^{1}\right\}$ is the original time sequence. The length of each coarse-grained time series is equal to the length of the original time series divided by the scale factor, $\tau$.

Here we consider time series with 5000 points and coarse-grain them up to scale 20 , so that the shortest time series has 250 points.

We then calculate an entropy measure (SampEn) for each coarse-grained time series plotted as a function of the scale factor $\tau$. We call this procedure multi-scale entropy (MSE). And at last we draw the corresponding function graph.

\section{Coupling Analysis of ECG and EMG based on Multiscale symbolic transfer entropy}

\subsection{Experimental data}

This paper uses ECG and EMG data in wake period and sleep time from MIT-BIH Polysomnographic Database, by PhysioBank. The database has multi-parameter data, including one for EEG and one for EMG. The sampling frequency is $512 \mathrm{~Hz}$, and it is recorded as long as 6 hours, noting every 30 seconds.

\subsection{Experimental method}

Based on the research above, we first extract 5000 points data in sleep time and wake period from one for EEG and one for EMG, from slep41. Calculating the TE value by multiscale symbolic transfer entropy, and then we know the coupling information between sleep time and wake period, and finally we analyze the result with comparison.

\subsection{Experimental result and analysis}

The result is shown as Fig. 2 and Fig. 3.

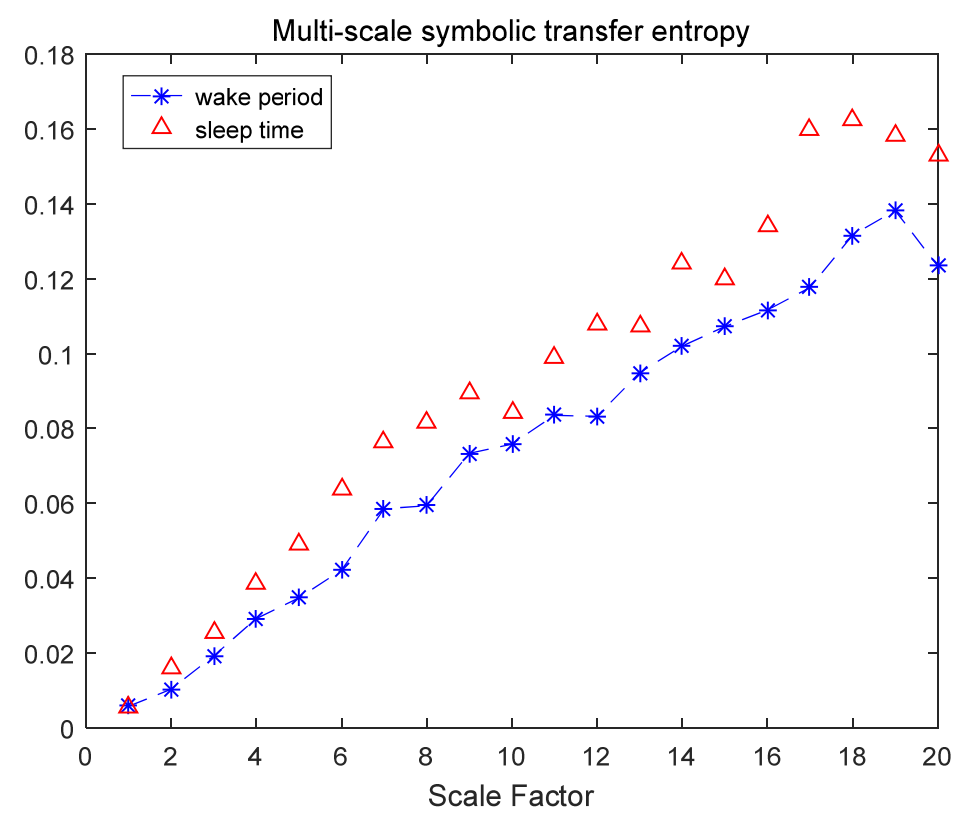

Fig. 2 Multiscale symbolic transfer entropy changes with scale factor changes 


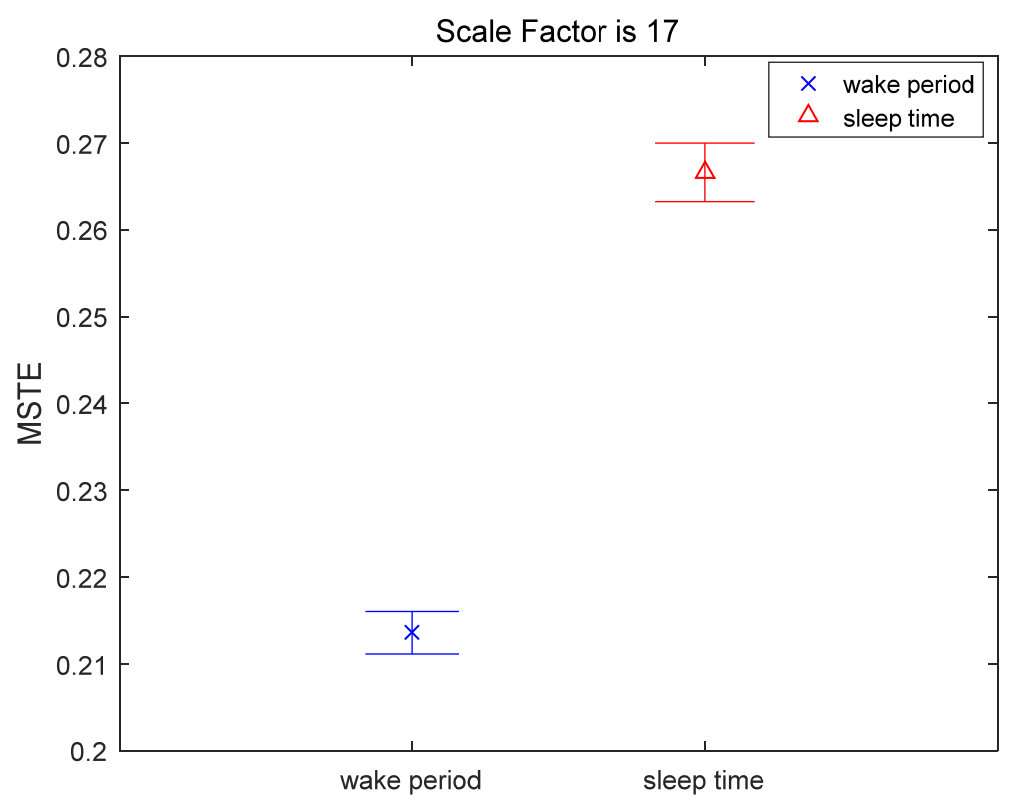

Fig. 3 the mean and variance of Multiscale symbolic transfer entropy

The experiment result shows that the value of it in the sleep time is larger than awake period, which means that the coupling of ECG and EMG is more significant in the sleep time, and we can capture the dynamic information in the signal and the change in system dynamics complexity more easily, which is better for the application of medical clinical practice. It can easily be seen that when the scale factor is 17 the difference is most obvious, and thus we do independent-samples $\mathrm{T}$ test for it. The result is shown in the Table 1.

\section{T-Test}

Group Statistics

\begin{tabular}{|cc|l|l|l|l|}
\hline \multicolumn{2}{|c|}{ VAR00033 } & $\mathrm{N}$ & Mean & Std. Deviation & Std. Error Mean \\
\hline VAR00032 & 1.00 & 16 & .21358154 & .049416782 & .012354196 \\
& 2.00 & 16 & .26661168 & .058020850 & .014505212 \\
\hline
\end{tabular}

Independent Samples Test

\begin{tabular}{|c|c|c|c|c|c|}
\hline & \multicolumn{4}{|c|}{ Levene's Test for Equality of Variances|t-test for Equality of Means } \\
\hline & & $F$ & S. & $t$ & df \\
\hline VAR00032 & $\begin{array}{l}\text { Equal variances assumed } \\
\text { Equal variances not assumed }\end{array}$ & .162 & .690 & $\begin{array}{l}-2.783 \\
-2.783\end{array}$ & $\begin{array}{l}30 \\
29.259\end{array}$ \\
\hline
\end{tabular}

Independent Samples Test

\begin{tabular}{|ll|l|l|l|}
\hline \multirow{2}{*}{} & & \multicolumn{3}{|l|}{ t-test for Equality of Means } \\
\cline { 2 - 5 } & & Sig. (2-tailed) & Mean Difference & $\begin{array}{l}\text { Std. } \\
\text { Difference }\end{array}$ \\
\hline VAR00032 & Equal variances assumed & .009 & -.053030145 & .019053276 \\
& Equal variances not assumed & .009 & -.053030145 & .019053276 \\
\hline
\end{tabular}

Independent Samples Test
\begin{tabular}{|ll|l|l|}
\hline \multirow{5}{*}{} & t-test for Equality of Means \\
\cline { 2 - 4 } & $\begin{array}{l}95 \% \text { Confidence Interval of the } \\
\text { Difference }\end{array}$ \\
\cline { 2 - 4 } & Lower & Upper \\
\hline VAR00032 & Equal variances assumed & -.091942126 & -.014118164 \\
& Equal variances not assumed & -.091983497 & -.014076793 \\
\hline
\end{tabular}

Table 1 the result of independent-samples $\mathrm{T}$ test

So, the value of Equal Variances Assumed is that $\mathrm{F}$ is 0.162 , and the probability is 0.690 , thus we have to read data from the first line, and the result is $t$ is -2.783 , (2-tailed) is 0.009 , far more smaller than 0.05 . The conclusion can be drawn that the value is larger in sleep time than in the wake period 
based on the multiscale symbolic transfer entropy, and the coupling between ECG and EMG is more obvious.

\section{Conclusions}

Based on the traditional transfer entropy, we use multiscale symbolic transfer entropy to analyze the coupling of ECG and EMG and only find that the multi-scale symbol transfer entropy value of it corresponds to different distribution trends at different scales and when the scale factor is 17 , the difference is most obvious. Then the analysis is based on the scale factor is 17 . Comparing with the traditional transfer entropy, this method can not only preserve the dynamics but also can assure that the consequences are not affected and thus can effectively analyze the non-stationary of the time sequence. The experiment shows that the value is larger in sleep time than in the wake period based on the multiscale symbolic transfer entropy, and the coupling between ECG and EMG is more obvious. Therefore, this method can helps us capture the dynamic information in the signal and the change in system dynamics complexity more easily, which is better for the application of medical clinical practice.

\section{Acknowledgments}

Project supported by the project is supported by the National Natural Science Foundation of China (Grant Nos. 61271082, 61401518, 31671006), Jiangsu Provincial Key R \& D Program (Social Development) (Grant No.BE2015700, BE2016773), the Natural Science Foundation of Jiangsu Province (Grant No. BK20141432),Natural Science Research Major Program in Universities of Jiangsu Province (Grant No.16KJA310002).

\section{References}

[1]Fang X L, Jiang Z L 2007 Acta Phys. Sin. 567330 (in Chinese)

[2] Meng Q F, zhou W, Chen Y H, Peng Y H 2010 Acta Phys. Sin. 59 123(in Chinese)

[3] Ma Q L, Bian C H, Wang J 2010 Acta Phys. Sin. 59 4480-4484 (in Chinese)

[4] Wang J, Ma Q L 2008 Chin. Phys. B 174424

[5] Hsu W Y 2012 Int. J. Neural Syst. 2251

[6] Petrantonakis P C, Hadjileontiadis L J 2012 IEEE Ton Signal Proc. 602604

[7] Thatcher R W 2012 Dev. Neuropsychol. 37476

[8] Wang J, Yu Z F 2012 Chin. Phys. B 21018702

[9] Musselman M, Djurdjanovic D 2012 Expert Systems with Appl. 3911413

[10] Wang R F, Zhang J H, Zhang Y, Wang X Y 2012 Biomed. Signal Proc. and Control 7490

[11] Orhan U, Hekim M, Ozer M 2012 J. of Med. Syst. 362219

[12] Acharya U R, Sree S V, Alvin A P C, Suri J S 2012 Expert Syst. Appl.39 9072

[13] Siuly S, Li Y 2012 IEEE T on Neur. Sys. and Reh. Eng. 20526

[14] Acharya U R, Molinari F, Sree S V, Chattopadhyay S, Ng K H, Suri JS 2012 Biomed. Signal Proc. and Control 7401

[15] Rosenblum M G, Pikovsky A S 2001 Phys. Rev. E 64045202

[16] Smirnov D A, Bezruchko B P 2003 Phys. Rev. E 68046209

[17] Smirnov D A, Bodrov M B, Velazquez J L P, Wennberg R A, Bezruchko B P 2005 Chaos 15 024102 
[18] Staniek M, Lehnertz K 2007 Phys. Rev. Lett. 99204101

[19] Shen W, Wang J 2010 Acta Phys. Sin. 60118702 (in Chinese)

[20] Thomas Schreiber. Measuring Information Transfer[J]. Phys RevLett,2000:461463

[21]Costa M, Goldberger AL, and Peng CK. Phys. Rev. Lett., Multiscale entropy analysis of complex physiologic time series, 2002; 89: 068102-1; 